\title{
Instrumentos mais utilizados na avaliação da exposição a Experiências Adversas na Infância: uma revisão da literatura
}

\author{
Most frequently used instruments for the assessment of exposure to \\ Adverse Childhood Experiences: a literature review
}

Flávia Garcia Pereira', Maria Carmen Viana'

DOI: $10.1590 / 0103-1104202112919$

RESUMO O objetivo deste estudo foi identificar e descrever os instrumentos mais frequentemente utilizados nas pesquisas epidemiológicas para a avaliação das Experiências Adversas na Infância, nos últimos dez anos. Trata-se de uma revisão da literatura, cujos critérios de inclusão foram artigos disponíveis na íntegra, nos idiomas inglês, espanhol e português, publicados e indexados nas bases de dados Medline e Lilacs, que citassem no resumo os instrumentos de avaliação utilizados nos estudos. Foram analisados 6 dos 38 instrumentos identificados nos 253 artigos selecionados para análise. Os instrumentos mais citados foram: 1) Childhood Trauma Questionnaire, 2) Childhood Experience of Care and Abuse, 3) Adverse Childhood Experiences Questionnaire, 4) Child Abuse and Trauma Scale, 5) Early Trauma Inventory Self Report e 6) Adverse Childhood Experiences International Questionnaire. Os instrumentos descritos diferiram quanto às propriedades psicométricas, à idade para aplicação e à quantidade de Experiências Adversas na Infância avaliadas. Predominaram estudos publicados em periódicos internacionais na língua inglesa. Três instrumentos apresentam versão em português vigente no Brasil, sendo que um deles considera somente a avaliação de aspectos específicos de Experiências Adversas na Infância, enquanto que os outros dois avaliam, também, outras experiências traumáticas.

PALAVRAS-CHAVE Inquéritos e questionários. Acontecimentos que mudam a vida. Adultos sobreviventes a eventos adversos na infância. Maus-tratos infantis.

ABSTRACT This study aimed to identify and describe the most frequently used instruments in epidemiological research to assess Adverse Childhood Experiences, in the past ten years. This is an integrative literature review, whose inclusion criteria were: full text articles in English, Spanish and Portuguese, published and indexed in the Medline and Lilacs databases, which cited in the summary the assessment instruments used in the studies. Six of the 38 instruments identified in the 253 articles selected for analysis were analyzed. The most cited instruments were: 1) Childhood Trauma Questionnaire, 2) Childhood Experience of Care and Abuse, 3) Adverse Childhood Experiences Questionnaire, 4) Child Abuse and Trauma Scale, 5) Early Trauma Inventory Self Report and 6) Adverse Childhood Experiences International Questionnaire. The instruments described differed in terms of psychometric properties, age for application and number of Adverse Childhood Experiences assessed. Studies published in international journals in the English language predominated. Three instruments have a Brazilian Portuguese version, one of which only considers the assessment of specific aspects of Adverse Childhood Experiences, while the other two also evaluates other traumatic experiences.

1 Universidade Federal do Espírito Santo (Ufes) Vitória (ES), Brasil. flaviagarciapereira1@gmail. com
KEYWORDS Surveys and questionnaires. Adverse childhoold experiences. Adult survivors of childhood trauma. Child maltreatment. 


\section{Introdução}

A exposição a Experiências Adversas na Infância (EAI) tem sido frequentemente documentada em diversos países ${ }^{1-6}$ e em todos os contextos geopolíticos e sociodemográficos ${ }^{7}$.

As EAI são definidas como quaisquer tipos de perpetrações de violência ou atos de omissão dirigidos à criança, além de um conjunto de situações que podem tornar disfuncional o ambiente familiar ${ }^{8}$. A partir do estudo Adverse Childhood Experiences (ACE), as EAI foram agrupadas em cinco tipos de violência envolvendo diretamente a criança (abuso físico, abuso sexual, abuso emocional, negligência física e negligência emocional) e cinco tipos relacionadas ao ambiente familiar disfuncional (uso/abuso de álcool e drogas, doença mental ou suicídio, violência, atividades criminais e separação ou divórcio parental), ocorrendo no contexto domiciliar da criança durante o seu desenvolvimento ${ }^{9,10}$.

As EAI podem ocasionar danos emocionais, lesões agudas com risco aumentado de morte imediata e incapacitação, e têm se mostrado significativamente associadas tanto à ocorrência de agravos à saúde física e mental na vida adulta quanto ao envolvimento de comportamentos de risco na adolescência ${ }^{2,8,9}$. Esta associação pode ser compreendida pela maior vulnerabilidade emocional durante a infância, quando a exposição a experiências adversas pode comprometer o equilíbrio necessário para o desenvolvimento saudável ${ }^{8}$, pois o estresse precoce e contínuo associado a tais eventos é capaz de alterar a função do eixo hipotálamo-hipófise-adrenal11,12. Embora respostas agudas deste eixo ao estresse sejam geralmente adaptativas, as respostas excessivas em conjunto com os genes de suscetibilidade para doenças podem levar a efeitos deletérios, a longo prazo"1.

Nessa perspectiva, a exposição às EAI tem consolidado o importante papel da própria infância no desenvolvimento humano, considerando as vivências pessoais como contínuas e cumulativas, resultantes de interações internas e externas, passadas e presentes ${ }^{13}$.
A partir da década de 1990, esse tema passou a despertar maior interesse entre pesquisadores, profissionais de saúde pública e defensores dos direitos humanos, visando à prevenção de maus-tratos infantis ${ }^{9,10}$, uma vez que o estudo ACE, além de demonstrar que os indivíduos com histórico de exposição a EAI apresentaram maior risco de doença coronariana, diabetes, câncer, acidente vascular cerebral e doença pulmonar obstrutiva crônica, entre outros males, na vida adulta ${ }^{9}$. Também evidenciou que a multimorbidade é mais frequente, a capacitação ocupacional é pior e a expectativa de vida é menor ${ }^{14-16}$.

Dado o impacto individual que as EAI podem imprimir ao longo da vida e os custos sociais envolvendo a sobrecarga dos sistemas de saúde e a perda de produtividade, tornou-se necessário o desenvolvimento de métodos adequados de avaliação para estudar os eventos adversos ocorridos na infância. Alguns instrumentos foram propostos nas últimas três décadas, inicialmente, com restrições quanto ao número de EAI avaliadas (apenas um ou dois tipos, como o abuso físico ou sexual) ${ }^{\mathbf{1 7}, 18}$, além de haver poucos estudos subsequentes avaliando suas propriedades psicométricas. Instrumentos com tais características dificultam a avaliação, tanto do efeito isolado de cada tipo de experiência quanto do efeito conjunto de múltiplos tipos de EAI ${ }^{19}$. Aos poucos, foram desenvolvidos novos instrumentos, incorporando outros tipos de EAI, sendo conduzidas avaliações sistemáticas de validade e confiabilidade. Assim, foram produzidas aproximações mais consistentes da realidade pregressa ${ }^{19}$. No entanto, com o aumento da disponibilidade de novas alternativas de avaliação, que pudessem ser mais adequadas a cada contexto estudado, o uso de instrumentos diferentes passou a dificultar as comparações entre países diversos e entre períodos diferentes da mesma população, ainda que abarcassem os mesmos construtos teóricos.

Em 2009, a Organização Mundial da Saúde (OMS), em parceria com o Centro de Controle de Doenças (CDC), elaborou o 
Adverse Childhood Experiences International Questionnaire (ACE-IQ), com o intuito de produzir um instrumento padronizado para avaliação e vigilância das EAI, mundialmente, possibilitando comparações transnacionais e embasando a formulação de programas e políticas de prevenção ${ }^{\mathbf{2 0}, 21}$.

Tendo em vista a crescente necessidade de estudar a exposição a EAI e a diversidade de instrumentos disponíveis para este fim, o objetivo do presente artigo foi identificar e descrever, através de uma revisão da literatura, os instrumentos mais utilizados na avaliação das EAI em investigações epidemiológicas realizadas nos últimos dez anos, desde a publicação do ACE-IQ.

\section{Metodologia}

A questão norteadora para a condução desta revisão foi assim definida: 'Diante da preocupação mundial acerca das possíveis consequências que as EAI podem acarretar na vida adulta, quais são os instrumentos de avaliação que têm sido mais utilizados nas pesquisas epidemiológicas realizadas nos últimos dez anos, sobre essa temática?'. Assim, este estudo se deu em duas etapas complementares: a busca sistemática de artigos publicados em periódicos indexados e a análise dos instrumentos mais frequentemente utilizados nesses estudos.

O levantamento e a análise dos artigos que compõem esta revisão da literatura foram executados no período de maio a dezembro de 2019.

Foram estabelecidos como critérios de inclusão para a seleção de artigos todos aqueles que: citassem no corpo do resumo os instrumentos utilizados para a avaliação das EAI; estivessem disponíveis em modo texto completo em português, inglês ou espanhol; e tivessem sido publicados nos últimos dez anos. Além disso, foram incluídos todos os artigos que utilizaram o ACE-IQ proposto pela OMS, considerando a relevância da tentativa de padronização de um instrumento abrangente, desenvolvido para ser utilizado em diferentes populações e culturas.
O Sistema Latino-Americano e do Caribe de Informação em Ciências da Saúde (Lilacs) e a Medical Literature Analysis and Retrieval System Online (Medline) foram as bases de dados utilizadas para a busca dos artigos científicos. Nessa etapa, foram utilizados os Descritores em Ciências da Saúde (DeCS) combinados com operadores boleanos: "child neglect" OR "life change events" OR "child abuse, adult survivors" OR "adult survivors of child abuse" OR "adult survivors of childhood trauma" OR "adult survivors, child abuse AND questionnaire". Em seguida, para um refinamento da busca, a seleção foi delimitada aos artigos que apresentavam as palavras "child" OR "childhood" no título.

Foi, então, realizada a análise crítica dos artigos identificados através da leitura dos resumos, e foram excluídos aqueles que não preencheram os critérios de inclusão, bem como artigos duplicados, além de revisões bibliográficas, dissertações, teses, pôsteres e estudos indisponíveis. Também foram excluídos instrumentos que não avaliavam as EAI vivenciadas pelo próprio entrevistado durante a sua infância, ou aqueles que foram preenchidos por pais ou responsáveis, professores e profissionais de saúde ou da justiça.

Os instrumentos que apareceram em quatro ou mais estudos foram analisados e descritos quanto às suas características, incluindo métodos de aplicação, idade mínima, abrangência de categorias e número de EAI avaliadas, escores globais, pontos de corte preconizados e propriedades psicométricas, desde que disponíveis na literatura geral. Para essa descrição, foi necessária uma busca complementar acerca das características dos instrumentos avaliados, guiada pelas referências bibliográficas contidas nos artigos incluídos nesta revisão.

\section{Resultados e discussão}

Foram encontrados 5.453 artigos, sendo 5.381 na base de dados Medline, e 72 no Lilacs. Após o refinamento da busca pelas palavras "child" OR 
"childhood" presentes no título, foram identificados 1.414 artigos na Medline e 25 no Lilacs, além de outros 19 estudos que utilizaram o ACE-IQ. Dentre os 1.439 artigos identificados nas bases de dados, foram excluídos: 563 indisponíveis; 579 que não se enquadravam na temática e/ou não indicavam, no resumo, os instrumentos de avaliação de EAI utilizados; 24 em outros idiomas (alemão-13; chinês - 7; francês - 2; húngaro - 1; e japonês - 1); e 20 duplicados. Foram, então, selecionados 253 artigos, sendo 252 na Medline e 1 no Lilacs, e avaliados através da leitura dos resumos e métodos, para identificação e descrição dos instrumentos de avaliação de EAI. O fluxograma do processo de busca, seleção e exclusão de artigos é apresentado na figura 1. Foram identificados 38 instrumentos utilizados na avaliação de EAI.

Figura 1. Protocolo de busca e resultado da revisão integrativa

Bases de dados eletrônicas onde foram realizadas as buscas: Medline e Lilacs.

Descritores de assunto e operadores boleanos utilizados na busca:

"child neglect" OR "life change events"

OR "child abuse, adult survivors" +

OR "adult survivors of child abuse"

OR "adult survivors of childhood trauma"

OR "adult survivors, child abuse

AND questionnaire".

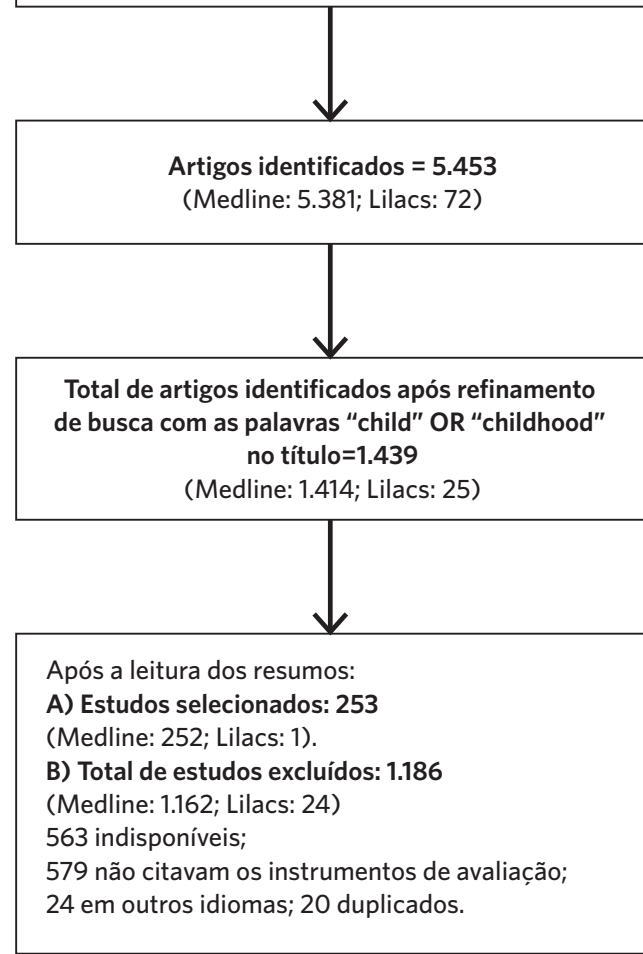

Instrumentos de avaliação de EAl identificados (número de artigos`)

1) Abuse Assessment Questionnaire (1)

2) Adverse Childhood Experiences Questionnaire (10)

3) Adverse Childhood Experiences-International Questionnaire (1)

4) Adverse Childhood Life Events (1)

5) Brief Child Abuse Potential Inventory (1)

6) Childhood Experience of Care and Abuse (15)

7) Centrality of Events Scale (1)

8) Child Abuse and Trauma Scale (4)

9) Child Abuse Potential Inventory (3)

10) Child Abuse Risk Assessment Scale (1)

11) Child Maltreatment Questionnaire (1)

12) Child Maltreatment Scales for Adults (1)

13) Child Neglect Questionnaire (1)

14) Childhood Abuse and Neglect Questionnaire (1)

15) Childhood Adversity Questionnaire (1)

16) Childhood Experiences of Violence Questionnaire (2)

17) Childhood History Questionnaire (3)

18) Childhood Physical and Sexual Abuse Questionnaire (1)

19) Childhood Trauma and Distress Scale (1)

20) Childhood Trauma Inteview (2)

21) Childhood Trauma Reports (1)

22) Child Interview Intervention (1)

23) Childhood Trauma Questionnaire (181)

24) Early Trauma Inventory Self-Report (4)

25) Early Life Stress Questionnaire (1)

26) Impact of Events Scale (1)

27) Interview or Traumatic Events in Childhood (2)

28) Juvenile Victimiation Questionnaire (2)

29) Lifetime Experiences Questionnaire (1)

30) List of Threatening Experiences Questionnaire (1)

31) Risky Families Questionnaire (3)

32) Sexual and Physical Abuse Questionnaire (1)

33) The Potentially Traumatic Experiences Questionnaire (1)

34) Trauma History Questionnaire (1)

35) Trauma Questionnaire (1)

36) Trauma Symptom Inventory (1)

37) Traumatic Antecedents Questionnaire (1)

38) Traumatic Life Events Questionnaire (2)

${ }^{\star} \mathrm{O}$ mesmo artigo pode ter citado dois instrumentos. 
Os instrumentos que apareceram em quatro ou mais estudos foram avaliados e descritos no quadro 1: 1) Childhood Trauma Questionnaire (CTQ-1994); 2) Childhood Experience of Care and Abuse Questionnaire (CECA.Q-1994); 3) Adverse Childhood Experiences Questionnaire (ACE questionnaire-1998); 4) Child Abuse and Trauma Scale (CATS-1995); 5) Early Trauma Inventory Self-Report (ETISF-2000); e 6) Adverse Childhood Experiences International Questionnaire (ACE-IQ-2009).

O CTQ foi o instrumento mais utilizado entre os artigos avaliados (181), conduzidos, em sua maioria, nos Estados Unidos e na Inglaterra, e publicados entre 2009 e 2017. Foi desenvolvido em 1994, com a proposição de 70 itens de avaliação ${ }^{17,19}$. Posteriormente, uma versão com 28 itens foi produzida, mantendo as mesmas propriedades psicométricas da versão original, com o objetivo de torná-lo uma ferramenta de aplicação mais rápida, com duração de aproximadamente cinco minutos ${ }^{19}$. As propriedades psicométricas do CTQ foram avaliadas através de sete amostras populacionais. A média da consistência interna, avaliada através do Alpha de Cronbach, obteve o valor mais alto para a variável de abuso sexual $(0,92)$ e o mais baixo para a negligência física $(0,66)$. Em uma avaliação da confiabilidade por meio de teste-reteste, entre adultos usuários de substâncias psicoativas, o coeficiente intraclasse variou de 0,79 a 0,81 , para negligência física e abuso sexual, respectivamente ${ }^{17}$. A versão original do CTQ demonstrou alta validade de construto, com índices de sensibilidade e especificidade próximos a 0,80 , em um estudo com 190 adolescentes com transtornos psiquiátricos ${ }^{17}$. O CTQ avalia cinco categorias de EAI (abuso físico, abuso sexual, abuso emocional, negligência física e negligência emocional) e tem sido utilizado em pesquisas clínicas e forenses ${ }^{22}$. Uma versão em português vigente no Brasil, denominada Questionário Sobre Traumas na Infância (Quesi), foi adaptada por Grassi-Oliveira ${ }^{23,24}$.

O CECA.Q é resultante do CECA inter$v_{i e w}{ }^{25}$. Foi utilizado em 15 estudos conduzidos em países da Europa, publicados entre 2009 e 2016. O instrumento avalia: 1) Cuidados parentais: antipatia e negligência; 2) Abuso físico; 3) Abuso sexual; e 4) Experiências sexuais. Apresenta confiabilidade (teste-reteste) variando de 0,5 a $0,84^{26}$.

O ACE questionnaire foi o terceiro instrumento mais usado (dez artigos) entre os que foram avaliados nesta revisão, publicados entre 2010 e 2016, e, em sua maioria, conduzidos nos Estados Unidos. O instrumento está contido no Family Heath History, que é um questionário mais amplo e apresenta outras variáveis, destinado a investigar a saúde geral da população americana ${ }^{27}$. O ACE questionnaire, desenvolvido para utilização no estudo sobre EAI, também conhecido como ACE study ${ }^{9}$, foi realizado em San Diego (EUA), no período de 1995 a 1997. Considerado como uma das mais robustas investigações acerca da exposição a situações de abuso e negligência, na infância, e seu impacto na saúde e no bem-estar, na vida adulta, seus resultados têm incentivado pesquisas em diferentes países e influenciado políticas públicas mundialmente ${ }^{9,10}$. Elaborado com base em outros instrumentos, como a Conflicts Tactics Scale e a National Health Interview Survey ${ }^{9}$, o ACE questionnaire apresenta possibilidade de avaliação de dez EAI de ocorrência no âmbito domiciliar, mensurando a violência direta contra a criança ou a exposição a adversidades advindas de disfunção familiar: 1) Abuso psicológico; 2) Abuso físico; 3) Abuso sexual; 4) Uso/abuso de álcool e drogas; 5) Doença mental ou suicídio; 6) Violência contra a mãe; 7) Envolvimento com atividades criminais; 8) Divórcio ou separação parental; 9) Negligência física; e 10) Negligência emocional 9 . O instrumento já foi adaptado para o português vigente em Portugal, recebendo o nome de Questionário da História de Adversidade na Infância ${ }^{\mathbf{2 8}}$.

O Child Abuse and Trauma Scale (CATS, Sanders and Becker-Lausen, 1995) foi usado em quatro artigos, todos realizados no Japão e publicados entre 2014 e 2016. O instrumento apresenta propriedades psicométricas 
consideradas como de nível satisfatório a excelente (Alpha de Cronbach de 0,63-0,90; teste-reteste $\mathrm{r}$ 0,71-0,91) ${ }^{29}$. O instrumento foi projetado para ser usado em um contexto de pesquisa epidemiológica, particularmente na avaliação de medidas em grupos populacionais, mas também pode ser útil, na avaliação clínica, como um instrumento de triagem ${ }^{29}$.

O Early Trauma Inventory Self-Report (ETISF-2000) é uma entrevista semiestruturada, com 52 itens agrupados em quatro domínios: abuso físico, sexual, emocional e experiências traumáticas gerais. Foi utilizado em quatro estudos de língua inglesa publicados entre 2011 e 2014. O ETISF deve ser aplicado por profissionais de saúde com experiência em atendimento a vítimas de violência ou por pessoas supervisionadas por um especialista da área ${ }^{30,31}$. Apresenta uma versão em português adaptada por Mello et al. ${ }^{31}$.

O Adverse Childhood Experiences International Questionnaire (ACE-IQ) foi citado em 20 artigos, majoritariamente na Ásia e na África, publicados entre 2014 e 2019. Foi elaborado com base em outros dois instrumentos: o ACE questionnaire e o módulo Adverse Childhood Experiences, do Behavioral Risk Factor Surveillance System (módulo ACE BRFSS) ${ }^{32}$, apresentando 13 categorias de EAI: 1) Abuso sexual; 2) Abuso emocional; 3) Abuso físico; 4) Negligência emocional; 5) Negligência física; 6) Violência familiar; 7) Uso de álcool e drogas; 8) Doença mental ou suicídio; 9) Envolvimento em atividades criminais; 10) Separação ou divórcio parental; 11) Violência comunitária; 12) Violência coletiva; e 13) Bullying (OMS, 2011). O ACE-IQ é um instrumento relativamente novo e está em processo de adaptação transcultural em alguns países ${ }^{21}$. Apresenta uma versão em português-brasileiro, adaptada por Pereira e Viana ${ }^{33}$ e disponibilizada no site da $\mathrm{OMS}^{34}$.

Com exceção do CTQ, que pode ser aplicado a partir dos 12 anos de idade, e do CECA.Q, que tem foco na avaliação de eventos traumáticos antes dos 17 anos, os demais instrumentos analisados foram desenvolvidos para aplicação em adultos ( $\geq 18$ anos), avaliando eventos adversos ocorridos nos primeiros 18 anos de vida. $\mathrm{O}$ ideal seria verificar a exposição a EAI o mais cedo possível, durante a infância, para estancar a violência e reduzir danos. A identificação de maus-tratos a menores de idade resulta em notificação compulsória na maioria dos países, devendo haver garantia de acesso a serviços de tratamento e medidas de proteção à criança e ao adolescente, que, via de regra, não estão no escopo da pesquisa epidemiológica e nem podem ficar sob a responsabilidade dos pesquisadores envolvidos ${ }^{20}$. Além disso, a notificação compulsória de violência e maus-tratos vigentes, rompendo o sigilo e o anonimato, tenderia a reduzir a busca por serviços de atenção, a adesão à avaliação e/ou a comprometer a fidedignidade das informações coletadas. Por estas razões, os instrumentos visam à investigação de histórico pregresso de exposição a adversidades na infância em indivíduos adultos. A identificação de maus-tratos infantis em andamento, bem como a implementação de medidas judiciais cabíveis e intervenções clínicas e psicológicas apropriadas devem ocorrer em outros âmbitos, que não os da pesquisa epidemiológica e clínica.

Quanto à forma de aplicação dos instrumentos descritos nesta revisão, três são autoaplicáveis (CTQ, CECA.Q, CATS), um deve ser aplicado através de entrevistas com um especialista na área (ETISF), um através de entrevistas face a face (ACE questionnaire) e o ACE-IQ pode ser autoaplicado ou administrado através de entrevistas face a face.

Quanto ao número de EAI avaliadas, os instrumentos variaram entre 4 e 13 tipos. $\mathrm{O}$ ACE-IQ é, entre os seis instrumentos analisados, o único que avalia situações adversas fora do âmbito domiciliar, como violência coletiva, violência comunitária e bullying. Outras classificações de EAI podem ser encontradas, de acordo com o interesse dos estudos, como é o caso do World Mental Health Surveys Consortium (WMH), que incluiu a adversidade econômica familiar à ocorrência de doenças físicas potencialmente letais na infância ${ }^{35}$. 
As definições e classificações de agressão à criança, assim como o entendimento sobre maus-tratos infantis, sofreram alterações ao longo da história, especialmente em relação ao papel educativo da punição física. A princípio, o termo 'maus-tratos infantis' atrelado à ocorrência de agressões físicas foi cedendo lugar ao termo 'abuso infantil' (1965), por compreender outras circunstâncias de aspectos familiares, econômicos e psicológicos, e, mais recentemente (na década de 1990), foi adotado o termo 'experiências adversas na infância',9,36.

Os instrumentos estão organizados com questões de respostas tipo Likert ou mescladas, Likert e dicotômicas.

As informações sobre características psicométricas e escores globais estão detalhadas no quadro 1.

A escolha de um instrumento adequado, seja para utilização em pesquisas epidemiológicas ou para avaliações clínicas, deve ponderar sobre os custos e benefícios decorrentes de sua aplicação, tendo em vista a população-alvo da pesquisa. É importante escolher um instrumento que permita atingir o objetivo do estudo, identificar se o método de aplicação é adequado à população a ser estudada e conseguir interpretar os resultados e as limitações do próprio instrumento, de forma a evitar erros sistemáticos advindos de fatores que interferem nas medidas avaliadas. A quantidade de itens, o tempo de aplicação e a necessidade de treinamento especializado (ex.: ETISF) também podem influenciar diretamente na escolha ou rejeição de um instrumento ${ }^{37}$. Além disto, alguns deles necessitam de autorização para a sua utilização, envolvendo custos adicionais, como é o caso do CTQ ${ }^{17}$. Apesar dessa exigência, o CTQ foi o instrumento mais citado nesta revisão. Ele apresenta a menor quantidade de itens, necessitando menor tempo para a sua aplicação, tem boas características psicométricas e abrange as EAI documentadas com maior impacto na saúde durante a infância e na vida adulta ${ }^{38}$, além de ser o instrumento mais antigo entre os descritos neste estudo.

Outro aspecto fundamental é a existência de informações psicométricas do instrumento.
Aqueles que já apresentam boa confiabilidade e validade, e que são satisfatórios em seus construtos, devem ser preferidos, ao invés de ser considerada a construção de um novo método de avaliação $0^{39}$. Mesmo que o instrumento escolhido apresente propriedades psicométricas satisfatórias, uma simples tradução - visto que a maioria dos instrumentos está em língua inglesa - é inapropriada e insuficiente para que ele possa ser usado ${ }^{37}$. Nesta perspectiva, uma adaptação transcultural deve ser implementada, para analisar questões referentes à linguagem, ao contexto cultural e ao estilo de vida da população-alvo, com o intuito de garantir equivalência conceitual e de itens, além de equivalência semântica, operacional e de mensuração entre o instrumento original e o adaptado $\mathbf{4 0 , 4 1}^{\text {. }}$

A variedade de instrumentos identificados nesta revisão (38) pode evidenciar a dificuldade de comparar as avaliações acerca das EAI entre países e justificar a necessidade de um instrumento padronizado, como é proposto pela OMS, através do ACE-IQ. A utilização do ACE-IQ tem como objetivo documentar e tornar comparáveis as ocorrências de eventos adversos mundialmente para, assim, melhor embasar decisões nas condutas administrativas, desdobradas em políticas públicas de enfrentamento e prevenção da violência ${ }^{20}$. Desta forma, o ACE-IQ apresenta-se como uma ferramenta sistemática para a coleta de dados e contém alguns requisitos exibidos em relatório pela OMS, tais como: boa acessibilidade (ser considerado como de fácil entendimento para a maior parte dos entrevistados $-85 \%$ ); baixa taxa de não resposta (20\%); maior abrangência de avaliação das EAI; e tempo de duração da entrevista relativamente curto, de 20 a 30 minutos ${ }^{20,21}$. O tempo de administração deve ser sempre considerado, quando se trata de um instrumento que será incluído em pesquisas de saúde mais amplas, uma vez que o principal objetivo na investigação das EAI é demonstrar suas associações com comportamentos de risco e/ou o desenvolvimento de doenças crônicas na vida adulta ${ }^{20,21,42}$. 
É possível que outros instrumentos estejam disponíveis na literatura, mas que não tenham sido apresentados neste artigo, por não terem se enquadrado nos critérios de busca. Além disto, este estudo apresenta como limitação a não inclusão dos módulos de avaliação de EAI contidos em grandes inquéritos populacionais, que geralmente incluem itens isolados de instrumentos já existentes.

Quadro 1. Características dos instrumentos de avaliação de EAI mais utilizados, identificados na revisão integrativa

\begin{tabular}{|c|c|c|c|}
\hline $\begin{array}{l}\text { Instrumento, autores e } \\
\text { ano de elaboração }\end{array}$ & $\begin{array}{l}\text { Países de publicação, } \\
\text { referências mais } \\
\text { recentes }\end{array}$ & $\begin{array}{l}\text { Número de questões e } \\
\text { categorias de EAl }\end{array}$ & Caracterização \\
\hline $\begin{array}{l}\text { 1) Childhood Trauma } \\
\text { Questionnaire (CTQ) } \\
\text { Bernstein DP, et al., 1994 } \\
\text { (original - } 70 \text { itens). } \\
\text { Bernstein DP, Fink, L, } \\
1998 \text { (28 itens). } \\
\text { Apresenta uma versão } \\
\text { em Português (Brasil) } \\
\text { adaptada por Grassi- } \\
\text {-Oliveira, R; Stein LM; } \\
\text { Pezzi, JC, } 2006 . \\
\text { Questionário sobre trau- } \\
\text { mas na infância (Quesi). }\end{array}$ & $\begin{array}{l}\text { Estados Unidos (65); } \\
\text { Inglaterra (63); Irlanda } \\
\text { (22); Holanda (15); } \\
\text { Alemanha (4); Brasil } \\
\text { (4); Austrália (3); Suíça } \\
\text { (3); Áustria (2). } \\
\text { Vicent J, et al., 2017. } \\
\text { Schwaninger PV, et al., } \\
2017 . \\
\text { Utzinger LM, et al., } \\
2016 . \\
\text { Vares EA, et al., } 2016 .\end{array}$ & $\begin{array}{l}28 \text { itens categorizados em: } \\
\text { 1) Abuso físico ( } 5 \text { itens); } \\
\text { 2) Abuso emocional ( } 5 \text { itens); } \\
\text { 3) Abuso sexual ( } 5 \text { itens); } \\
\text { 4) Negligência física ( } 5 \text { itens); } \\
\text { 5) Negligência emocional ( } 5 \\
\text { itens). } \\
\text { Apresenta três itens reversos. }\end{array}$ & $\begin{array}{l}\text { Ferramenta de triagem, autoaplicável. } \\
\text { Avalia a ocorrência de um ou mais eventos traumáticos antes } \\
\text { dos } 10 \text { anos. Desenvolvido para ser aplicado em adolescentes } \\
\text { (a partir de } 12 \text { anos) e adultos. } \\
\text { Cada item é avaliado de acordo com escala Likert de } 5 \text { pontos } \\
\text { (1=nunca; } 2=\text { poucas vezes; } 3=\text { às vezes; } 4=\text { muitas vezes; } \\
\text { 5=sempre). } \\
\text { Cada categoria de adversidade é composta por cinco asserti- } \\
\text { vas, com escores variando de } 5 \text { a } 25 \text {. } \\
\text { EAl são classificadas em: nenhuma, leve, moderada ou grave, } \\
\text { de acordo com a pontuação. } \\
\text { Alpha de Chronbach entre 0,60 e 0,96. Correlação teste- } \\
\text {-reteste: Correlaç̃es de Pearson entre 0,79 e 0,86, em todas } \\
\text { as subescalas (Bernstein DP, et al., 1994). }\end{array}$ \\
\hline $\begin{array}{l}\text { 2) Childhood Experience of } \\
\text { Care and Abuse (CECA.Q) } \\
\text { Oriundo do CECA inter- } \\
\text { view (Bifulco A, et al., } \\
\text { 1994). }\end{array}$ & $\begin{array}{l}\text { Estados Unidos (5); } \\
\text { Holanda (5); Inglaterra } \\
\text { (2); Alemanha (1); Brasil } \\
\text { (1); Irlanda (1). } \\
\text { Infurna MR, et. al., } 2015 . \\
\text { Trauelsen AM, et al., } \\
2015 . \\
\text { Gayer-Anderson C, et } \\
\text { al., } 2015 \text {. } \\
\text { Theleritis C, et al., } 2014 .\end{array}$ & $\begin{array}{l}32 \text { itens categorizados em: } \\
\text { 1) Cuidados parentais: antipatia e } \\
\text { negligência ( } 16 \text { itens); } \\
\text { 2) Abuso físico ( } 5 \text { itens); } \\
\text { 3) Abuso sexual ( } 3 \text { itens); } \\
\text { 4) Experiências sexuais ( } 8 \text { itens). }\end{array}$ & $\begin{array}{l}\text { Autoaplicável. } \\
\text { Avalia a ocorrência de um ou mais eventos traumáticos antes } \\
\text { dos } 17 \text { anos. } \\
\text { Os itens são avaliados em escala Likert (sim/definitivamen- } \\
\text { te=5 ou 4; incerto=3; não/de jeito nenhum=2 ou 1) ou ques- } \\
\text { tões dicotômicas (sim=1; não=0). Outros tipos de itens. } \\
\text { Consistência interna: Alpha de Chronbach=0,81 para avalia- } \\
\text { ção de antipatia; Alpha de Cronbach=0,80 para a avaliação } \\
\text { de negligência. Confiabilidade (teste-reteste): r=0,84 negli- } \\
\text { gência materna; r=0,5 negligência paterna; r=0,74 antipatia } \\
\text { materna; r=0,71 antipatia paterna; r=0,52 abuso físico } \\
\text { provocado pela mãe; r=0,51 abuso físico provocado pelo pai; } \\
\text { r=0,70 para os itens de triagem de abuso sexual; e r=0,61 } \\
\text { para os itens de gravidade sobre abuso sexual (Bifulco A, et } \\
\text { al., 2005). }\end{array}$ \\
\hline $\begin{array}{l}\text { 3) Adverse Childhood } \\
\text { Experiences Questionnaire } \\
\text { (ACE questionnaire) } \\
\text { Felliti VJ, et al., } 1998 . \\
\text { Apresenta uma versão } \\
\text { portuguesa (Portugal) } \\
\text { adaptada por Silva e } \\
\text { Maia, } 2006 . \\
\text { Questionário da História } \\
\text { de Adversidade na In- } \\
\text { fância. }\end{array}$ & $\begin{array}{l}\text { Estados Unidos (6); } \\
\text { Inglaterra (3); Alema- } \\
\text { nha (1). } \\
\text { Glowa, PT; Olson, AL; } \\
\text { Johnson, DJ, } 2016 . \\
\text { Jião-Mei, X; Wen, G; } \\
\text { Feng-Lin, } 2016 . \\
\text { Liu, Z, et al., } 2016 . \\
\text { Skjothaug T, et al., } 2015 .\end{array}$ & $\begin{array}{l}32 \text { itens categorizados em: } \\
\text { 1) Abuso psicológico ( } 3 \text { itens); } \\
\text { 2) Abuso físico ( } 4 \text { itens); } \\
\text { 3) Abuso sexual ( } 4 \text { itens); } \\
\text { 4) Uso/abuso de álcool e drogas } \\
\text { ( } 3 \text { itens); } \\
\text { 5) Doença mental ou suicídio ( } 2 \\
\text { itens); } \\
\text { 6) Violência contra a mãe ( } 4 \text { itens); } \\
\text { 7) Envolvimento com atividades } \\
\text { criminais ( } 1 \text { item); } \\
\text { 8) Divórcio ou separação parental } \\
\text { (1 item); } \\
\text { 9) Negligência física ( } 5 \text { itens); } \\
\text { 10) Negligência emocional (5 } \\
\text { itens). }\end{array}$ & $\begin{array}{l}\text { Avalia a ocorrência de um ou mais eventos traumáticos } \\
\text { antes dos } 18 \text { anos (autorrelato). } \\
\text { Os itens são avaliados em escala Likert de } 0 \text { a } 4 \text { ( } 0=\text { nun- } \\
\text { ca; } 1=\text { uma vez, } 2=\text { duas vezes; } 3=\text { frequentemente; } 4=\text { mui- } \\
\text { tíssimas vezes) e em questões dicotômicas ( sim=1 ou } \\
\text { não=0). Escore de } 0 \text { a } 10 \text { pontos. } \\
\text { Teste-reteste: Coeficiente Kappa para abuso emocio- } \\
\text { nal=0,66; abuso físico=0,55; abuso sexual=0,69; uso/ } \\
\text { abuso de substâncias=0,75; violência interpessoal=0,77 } \\
\text { (Dube SR, et al., 2004; Ritacco G; Suffla L, 2012). }\end{array}$ \\
\hline
\end{tabular}




\section{Países de publicação, \\ Instrumento, autores e referências mais ano de elaboração}

4) Child Abuse and Trauma Scale (CATS)

Sanders B, Lausen EB, 1995.

5) Early Trauma Inventory Self-Report (ETISF) Bremner JD, et al., 2000. Apresenta uma versão em português brasileiro adaptada por Mello et al. (2010).

\section{6) Adverse Childhood}

Experiences International Questionnaire (ACE-IQ)

OMS, 2009.

OMS, 2011.

Apresenta uma versão em português brasileiro adaptada por Pereira e

Viana, 2020.

Experiências Adversas na Infância - Questionário Internacional (EAI-QI).

Hayashi Y, et al., 2015

\section{Número de questões e categorias de EAI}

Japão (4).

Toda H, et al., 2016.

Nakai Y, et al., 2015.

Nakai Y, et al., 2014. (16 itens);

3) Punição (5 itens);
38 itens categorizados em:

1) Negligência/ambiente familiar

2) Abuso sexual (6 itens);

4) Abuso emocional (6 itens);

5) Questões reversas (5 itens).

Inglaterra (2); Irlanda (1); Estados Unidos (1).

Plaza A, et al., 2014. Plaza A, et al., 2012. Jeon HJ, et al., 2012.

Powers AD, et al., 2011.

China (1)

Tran QA, et al., 2015.

Adicionados à revisão:

Arábia Saudita (5);

Tunísia (3); Bagdá (2);

China (2); Malawi (1);

Holanda (1); Quênia (1); Líbano (1); África do Sul (1); Nigéria (1); Coréia do Sul (1).
52 itens. Apresenta quatro domínios:

1) Abuso físico (9 itens);

2) Abuso sexual (11 itens);

3) Abuso emocional (8 itens);

4) Experiências traumáticas gerais (24 itens).

43 itens. Destes, 28 itens categorizam 13 EAl:

1) Abuso emocional ( 2 itens);

2) Abuso físico (2 itens);

3) Abuso sexual (4 itens);

4) Violência familiar (3 itens);

5) Uso/abuso de álcool e drogas (1 item):

6) Doença mental ou suicídio (1 item);

7) Envolvimento com atividades criminais (1 item);

8) Separação/divórcio parental, morte dos pais (2 itens);

9) Negligência física (3 itens);

10) Negligência emocional (2

itens);

11) Bullying (1 item);

12) Violência comunitária (3

itens);

13) Violência coletiva (4 itens).

\section{Caracterização}

Autoaplicável.

Avalia a ocorrência de um ou mais eventos traumáticos (não menciona o limite da idade; o instrumento inclui o termo "criança ou adolescente" no cabeçalho e nos itens; apenas um item relativo a abuso sexual considera idade inferior a 14 anos).

Os itens são avaliados em escala Likert de 0 a 4 ( $0=$ nunca; $1=$ raramente; 2 =às vezes; $3=$ frequentemente; $4=$ sempre).

Escore: as pontuações totais na medida são divididas pelo número de itens para produzir uma pontuação média. Uma pontuação maior significa maiores níveis de maus-tratos; alguns itens são codificados inversamente. Consistência interna: alpha=0,63 a 0,90; Confiabilidade (teste-reteste): $r=0,71$ a 0,91 (Sanders B, Lausen EB, 1995).

Avalia a ocorrência de um ou mais eventos traumáticos antes dos 18 anos. Desenvolvido para entrevistas realizadas por um clínico.

Os itens são avaliados de forma dicotômica (sim; não). Coeficiente de correlação teste-reteste de 0,91 ( $d f=9$; $p<0,001$ ), índice de correlação intraclasse entre avaliadores de 0,99 ( $F=157,44 ; d f=10,11 ; p<0,0001)$ e 0,95 de consistência interna (Bremner JD, et al., 2000).

Autoaplicável ou entrevistas face a face.

Avalia a ocorrência de um ou mais eventos traumáticos antes dos 18 anos.

Os itens sobre EAI estão divididos em escala Likert de 0 a 3( $0=$ nunca; $1=$ uma vez; $2=$ poucas vezes; $3=$ muitas vezes) e itens dicotômicos ( $\operatorname{sim}=1$; não=0).

Escore de 0 a 13.

Coeficiente Alpha de Chronbach para casamento, relacionamento com os pais/responsáveis, ambiente familiar, violência entre pares, testemunho de violência comunitária e exposição à guerra/violência coletiva $=0,65 ; 0,71$; 0,$72 ; 0,69 ; 0,81 ;$ e 0,79, respectivamente. (Kazeem OT, 2015).

Fonte: Elaboração própria. 


\section{Considerações finais}

O propósito deste estudo foi identificar os instrumentos frequentemente utilizados na avaliação de EAI, nos últimos dez anos, produzindo um material abrangente, disponível para sua utilização em estudos brasileiros ou de outros países de língua portuguesa. Estes achados podem ser complementados por revisões anteriores, que incluem outros tipos de instrumentos de avaliação das EAI, como é o caso da revisão sistemática publicada em 2004, abarcando estudos divulgados entre 1985 e $2003^{\mathbf{4 3}}$, ou outras subsequentes ${ }^{\mathbf{4 4}, 45}$.

Esta revisão da literatura resultou na identificação de uma variedade de instrumentos utilizados para uma avaliação sistemática das EAI. Entre os 38 encontrados, o Childhood Trauma Questionnaire foi o mais utilizado nas pesquisas epidemiológicas. Apenas três dos instrumentos avaliados apresentam versões em português vigente no Brasil: o Childhood Trauma Questionnaire, o Early Trauma Inventory Self Report e o Adverse Childhood Experiences-International Questionnaire.

A variedade de instrumentos encontrados e a análise dos que foram frequentemente utilizados evidenciam as discrepâncias nos métodos de avaliação das diferentes categorias de EAI, dificultando a comparabilidade entre estudos e a identificação acurada das estimativas de prevalência das EAI nas populações.

Tendo em vista o impacto que as EAI podem causar ao longo da vida, tanto no desenvolvimento de doenças crônicas quanto no engajamento em comportamentos de risco, e todas as possíveis consequências da exposição a elas na procura por atendimento médico adequado, o conhecimento prévio dos instrumentos de avaliação das EAI pode auxiliar na escolha mais adequada dos instrumentos disponíveis. Pode, ainda, ajudar na decisão de desenvolver um novo instrumento, caso nenhum dos existentes seja suficientemente específico e sensível ao propósito da avaliação em um contexto cultural diferente, no qual se pretenda realizar novas pesquisas ou empreender um processo de adaptação transcultural, quando um instrumento já desenvolvido é adequado ao que se propõe, mas ainda não está disponível.

\section{Colaboradoras}

Pereira FG (0000-0003-0197-511X)* e Viana MC (0000-0002-0464-4845)* contribuíram igualmente para a elaboração do manuscrito. 


\section{Referências}

1. Pinto VCP, Alves JFC, Maia AC. Adversidade na infância prediz sintomas depressivos e tentativas de suicídio em mulheres adultas portuguesas. Estud. Psicol. 2015; 32(4):617-625.

2. Almunnef M, Hollinshed D, Saleheen H, et al. Adverse childhood experiences and association with health, mental health, and risky behavior in the kingdom of Saudi Arabia. Child Abuse Negl. 2016; 60:10-17.

3. Campbell JA, Walker RJ, Egede LE. Associations between adverse childhood experiences, high-risk behaviors, and morbidity in adulthood. Am J Prev Med. 2016; 50(3):344-352.

4. Musa S, Peek-Asa C, Jovanovic N, et al. Association of adverse childhood experiences and health risk behaviors among Young adults visiting a regional primary healthcare center, Federation of Bosnia and Herzegovina. Plos One. 2018 [acesso em 2020 mar 10]; 13(3):1-14. Disponível em: https://journals.plos.org/ plosone/article?id=10.1371/journal.pone.0194439.

5. Naal H, El Jalkh T, Haddad R. Adverse childhood experiences in substance use disorder outpatients of a Lebanese addiction center. Psychol Health Med. 2018; 23(9):1137-44.

6. Viana MC, Lim CCW, Pereira FG, et al. Previous Mental Disorders and Subsequent Onset of Chronic Back or Neck Pain: Findings From 19 Countries. J Pain. 2018; 19(1):99-110.

7. Almuneef M, Qayad M, Aleissa M, et al. Adverse childhood experiences, chronic diseases, and risky health behaviors in Saudi Arabian adults: a pilot study. Child Abuse Negl. 2014; 38(11):1787-93.

8. Clemons JC, DiLillo D, Martinez IG, et al. Co-ocurring forms of child maltreatment and adjustment reported by Latina college students. Child Abuse Negl. 2003; 27(7):751-67.

9. Felitti VJ, Anda RF, Nordenberg D, et al. Relationship of abuse and household dysfunction to many of the leading causes of death in adults: the Adverse Childhood Experiences (ACE) Study. Am J Prev Med. 1998; 14(4):245-58.

10. Felitti VJ, Anda R. The relationship of adverse childhood experiences to adult medical diseade, psychiatric disorders and sexual behavior: implications for healthcare. In: Lanius RA, Vermetten E, Pain C. The impact of early trauma on health and disease: the hidden epidemic. Reino Unido: Cambridge University Press; 2010. p. 77-87.

11. Mello FM, Faria AA, Mello AF, et al. Maus-tratos na infância e psicopatologia no adulto: caminhos para a disfunção do eixo hipotálamo-pituitária-adrenal. Rev. Bras. Psiquiatr. 2009; 31(suplII):S41-8.

12. Silva S, Maia AC. Experiências adversas na infância e tentativas de suicídio em adultos com obesidade mórbida. Rev. psiquiatr. 2010; 32(3):69-72.

13. Alves J, Maia A. Experiências adversas durante a infância e comportamentos de risco para a saúde em mulheres reclusas. Psicol. Saúde doença. 2010; 11(1):151-171.

14. Bellis MA, Hughes K, Leckenby N, et al. Measuring mortality and the burden of adult disease associated with adverse childhood experiences in England: a national survey. J. Public Health (Oxf.). 2015; 37(3):44554.

15. Brown DW, Anda RF, Tiemeier H, et al. Adverse childhood experiences and the risk of premature mortality. Am J Prev Med. 2009; 37(5):389-96.

16. Metzler M, Merrick MT, Klevens J, et al. Adverse childhood experiences and life opportunities: Shifting the narrative. Child. Youth Serv. Rev. 2017; (72)141-149.

17. Bernstein DP, Fink L, Handelsman L, et al. Initial realiability and validity of a new retrospective measure of child abuse and neglect. Am J Psychiatry 1994; 151(8):1132-6. 
18. Montecinos REM, Añasco RC. Relación de las experiencias sexuales infanto-juveniles com la confianza diádica y el temor a la intimidad, em estudiantes universitarios. Ter Psicol. 2008 [acesso em 2020 mar 10]; 26(2):229-239. Disponível em: https://scielo.conicyt.cl/pdf/terpsicol/v26n2/art09.pdf.

19. Bernstein DP, Stein JA, Newcomb MD, et al. Development and validation of a brief screening version of Childhood Trauma Questionnaire. Child Abuse Negl 2003; 27:169-190.

20. World Health Organization. Addressing adverse childhood experiences to improve public health: expert consultation. 2009. [acesso em 2020 mar 10]. Disponível em: https://www.who.int/violence_injury_prevention/violence/activities/adverse_childhood_experiences/global_research_network/en/.

21. World Health Organization. Adverse childhood experiences international questionnaire. Piloty study review and finalization meeting. 2011 [acesso em 2020 mar 10]. Disponível em https://www.who.int/ violence_injury_prevention/violence/activities/adverse_childhood_experiences/global_research_network/en/.

22. Bernstein D, Fink L. Childhood Trauma Questionnaire: a retrospective self-report. San Antonio: The Psicological Corporation; 1998.

23. Brodski SK, Zanon C, Hutz CS. Adaptação e validação do questionário sobre traumas na infância (QUESI) para uma amostra não-clínica. Aval. Psicol. 2010; 9(3):499-501.

24. Grassi-Oliveira R, Stein LM, Pezzi JC. Tradução e validação de conteúdo da versão em português do Childhood Trauma Questionnaire. Rev Saúde Públ. 2006; 40(2):249-55.

25. Bifulco A, Brown GW, Harris TO. Childhood Experience of Care and Abuse (CECA): a retrospective interview measure. J Child Psychol Psychiatry. 1994; 35(8):1419-35.
26. Bifulco A, Bernazzani O, Moran PM. The childhood experience of care and abuse questionnaire (CECA.Q): validation in a community series. Br J Clin Psychol. 2005; 44(Pt4):563-81.

27. Centers for Disease Control and Prevention. Violence Prevention. [acesso em 2020 mar 10]. Disponível em: https://www.cdc.gov/violenceprevention/childabuseandneglect/acestudy/about.html.

28. Silva S, Maia A. Versão portuguesa do Family ACE Questionnaire. In: Noronha A, Machado C, Almeida L, et al., coordenadores. Actas da XIII Conferência Internacional de Avaliação Psicológica: Formas e Contextos. Braga: Psiquilibrios; 2008.

29. Sanders B, Becker-Lausen E. The measurement of psychological maltreatment: early data on the child abuse and trauma scale. Child Abuse Negl. 1995; 19:315-23.

30. Bremner JD, Vermetten E, Mazure CM. Development and preliminary psychometric properties of an instrument for the measurement of childhood trauma: the Early Trauma Inventory. Depress Anxiety. 2000; 12(1):1-12.

31. Mello MF, Schoedl AF, Cadrobbi M, et al. Adaptação transcultural e consistência interna do Early Trauma Inventory (ETI). Cad. Saúde Pública. 2010; 26(4):713724.

32. Centers for Disease Control and Prevention. Behavioral Risk Factor Surveillance System. [acesso em 2020 mar 10]. Disponível em: https://www.cdc.gov/ brfss/questionnaires/index.htm.

33. Pereira FG, Viana MC. Adaptação transcultural do Adverse Childhood Experiences - International Questionnaire. Rev Saúde Públ. No prelo, 2021.

34. World Health Organization. Adverse Childhood Experiences International Questionnaire (ACE-IQ). [acesso em 2021 jan 29]. Disponível em: https://www. who.int/publications/m/item/adverse-childhood-experiences-international-questionnaire-(ace-iq). 
35. Kessler RC, McLaughlin KA, Green JG, et al. Childhood adversities and adult psychopathology in the WHO World Mental Health Surveys. Br J Psychiatry. 2010; 197(5):378-85.

36. Oliveira RV, Pais LG. A origem dos maus-tratos: revisão sobre a evolução histórica das percepções de criança e maus-tratos. 2014 [acesso em 2020 mar 10]; 3(1):3649. Disponível em: http://repositorio.ispa.pt/bitstream/10400.12/3135/1/PCH_2014_3\%281\%29_36.pdf.

37. Gorenstein C, Wang Y-P. Fundamentos de mensuração em saúde mental. In: Gorenstein C, Wang Y-P, Hungerbühler I, organizadores. Instrumentos de avaliação em saúde mental. Porto Alegre: Artmed; 2016.

38. Dong M, Anda RF, Dube SR, et al. The relationship of exposure to childhood sexual abuse to other forms of abuse, neglect, and household dysfunction during childhood. Child Abuse Negl. 2003; 27(6):625-39.

39. Reichenheim ME, Moraes CL. Operacionalização de adaptação transcultural de instrumentos de aferição usados em epidemiologia. Rev Saúde Pública. 2007; 41(4):665-73.

40. Guillemin F, Bombardier C, Beaton D. Cross-cultural adaptation of health-related quelity of measures: literature review and proposed guidelines. J Clin Epidemiol. 1993; 46(12):1417-32.
41. Beaton DE, Bombardier C, Guillemin F, et al. Guidelines for the process of cross-cultural adaptation of self-reported measures. Spine 2000; 25(24):3186-91.

42. Almuneef M, ElChoueiry N, Saleheen HN, et al. Gender-based disparities in the impact of adverse childhood experiences on adult health: findings from a national study in the Kingdom of Saudi Arabia. Int J Equity Health. 2017; (16):90.

43. Roy CA, Perry C. Instruments for the assessment of childhood trauma in adults. J Nerv Ment Dis. 2004; 192(5):343-51.

44. Burgermeister D. Childhood adversity: a review of measurement instruments. J Nurs Meas. 2007; 15(3):16376.

45. Oh DL, Jerman P, Purewal, SKB. Review of Tools for Measuring Exposure to Adversity in Children and Adolescents. J Pediatr Health Care. 2018; 32(6):564583.

Recebido em16/04/2020

Aprovado em 05/11/2020

Conflito de interesses: inexistente

Suporte financeiro: Coordenação de Aperfeiçoamento de Pessoal

de Nível Superior (Capes) - Código de Financiamento 001 